\title{
BOUNDEDNESS OF MAXIMAL FUNCTIONS AND SINGULAR INTEGRALS IN WEIGHTED $L^{p}$ SPACES
}

\author{
JOSE L. RUBIO DE FRANCIA
}

\begin{abstract}
Given a weight $w(x)>0$ in $\mathbf{R}^{n}$, necessary and sufficient conditions are found for the boundedness of the Hardy-Littlewood maximal function and singular integral operators from $L^{p}(w)$ to some other weighted $L^{p}$ space. The dual question is also considered and partially answered.
\end{abstract}

1. Introduction. Weighted $L^{p}$ inequalities for the Hardy-Littlewood maximal function as well as for some singular integral operators are known to hold if and only if the weight function $w$ belongs to Muckenhoupt's class $A_{p}[8,2]$. In [9], the following question was raised: Find conditions on $w(x)$ so that these operators are bounded from $L^{p}(w)$ to some other weighted space $L^{p}(u)$. For the conjugate function operator on the torus T, P. Koosis [6] has found that a necessary and sufficient condition in the $L^{2}$ case is $w^{-1} \in L^{1}(\mathbf{T})$. Here we shall extend this result to $L^{p}$, where the condition becomes $w^{-p^{\prime} / p} \in L^{1}(T)$, and is the same for the conjugate function as for the Hardy-Littlewood maximal operator. Moreover, all this can be extended to $\mathbf{R}^{n}$, where, in the $L^{p}$ case, the weight $w$ must verify $w^{-p^{\prime} / p} \in L_{\text {loc }}^{1}$ with an additional condition limiting the growth at infinity of $w^{-p^{\prime} / p}$.

2. Boundedness of the maximal function. Let $M$ denote the Hardy-Littlewood maximal operator in $\mathbf{R}^{n}$

$$
M f(x)=\sup _{x \in Q} \frac{1}{|Q|} \int_{Q}|f|
$$

where $Q$ is always a cube in $\mathbf{R}^{n}$ and $|\cdot|$ denotes Lebesgue measure. We shall consider in particular the cubes $Q_{R}=\left\{x \in \mathbf{R}^{n}: \max _{1<i<n}\left|x_{i}\right| \leqslant R\right\} . L^{0}\left(\mathbf{R}^{n}\right)$ will be the space of all measurable functions in $\mathbf{R}^{n}$ provided with the topology of local convergence in measure, i.e. $\lim _{j} f_{j}=0$ (in $L^{9}$ ) iff $\lim _{j}\left|\left\{x \in Q:\left|f_{j}(x)\right|>\lambda\right\}\right|=0$ for every cube $Q$ and every $\lambda>0$. We recall that a pair $(v, w)$ of positive measurable functions in $\mathbf{R}^{n}$ belongs to the class $A_{p}, 1<p<\infty$, when

$$
\sup _{Q}|Q|^{-1}\left(\int_{Q} v\right)^{1 / p}\left(\int_{Q} w^{-p^{\prime} / p}\right)^{1 / p^{\prime}}<\infty
$$

and this condition is equivalent to the fact that $M$ be bounded from $L^{p}(w)$ to weak- $L^{p}(v)$ (see [9]).

Received by the editors December 19, 1980; presented to the Society, December 11, 1980.

1980 Mathematics Subject Classification. Primary 42B20, 42B25; Secondary 42A20, 42 A50.

Key words and phrases. Hardy-Littlewood maximal function, singular integral, weighted norm inequalities. 
TheOREM A. Given $w(x)>0$ in $\mathbf{R}^{n}$ and $1<p<\infty$, the following conditions are equivalent:

(a) There exists $u(x)>0$ in $\mathbf{R}^{n}$ such that $M$ is bounded from $L^{p}(w)$ to $L^{p}(u)$.

(b) $w^{-p^{\prime} / p} \in L_{\text {loc }}^{1}$ and $\lim \sup _{R \rightarrow \infty}\left|Q_{R}\right|^{-p^{\prime}} \int_{Q_{R}} w(x)^{-p^{\prime} / p} d x<\infty$.

(c) There exists $v(x)>0$ in $\mathbf{R}^{n}$ such that $(v, w) \in A_{p}$.

(d) $M f(x)<\infty$ a.e. for every $f \in L^{p}(w)$.

(e) $M$ is a continuous operator from $L^{p}(w)$ to $L^{0}\left(\mathbf{R}^{n}\right)$.

Proof. It is obvious that (c) implies (b). If (b) holds, for every $f \in L^{p}(w)$ we have by Hölder's inequality

$$
\underset{R \rightarrow \infty}{\lim \sup }\left|Q_{R}\right|^{-1} \int_{Q_{R}}|f| \leqslant\|f\|_{L^{p}(w)} \limsup _{R \rightarrow \infty}\left|Q_{R}\right|^{-1}\left(\int_{Q_{R}} w^{-p^{\prime} / p}\right)^{1 / p^{\prime}}<\infty
$$

and this is equivalent to $M f(x)<\infty$ a.e. Thus (d) is obtained from (b). An application of the Banach principle (see [4]) proves that (d) implies (e). Since $M$ is the maximal operator corresponding to a family of positive operators, it is a consequence of Nikishin's theorem (see [5]) that (e) implies (c). Therefore, (b), (c), (d) and (e) are equivalent, and (a) implies that $(u, w) \in A_{p}$. We only have to prove that (a) follows from (b), which is the main point of the theorem. For each fixed cube $Q$, we shall prove that there exists $u_{Q}(x)>0$ on $Q$ such that

$$
\int_{Q}(M f)^{p} u_{Q}<\int_{\mathbf{R}^{n}}|f|^{p_{w}} \quad\left(f \in L^{p}(w)\right) .
$$

Once this is done, it suffices to take a partition of $\mathbf{R}^{n}$ into a sequence $\left(Q_{j}\right)$ of disjoint cubes, and then (a) is verified with $u(x)=\Sigma_{j} 2^{-j} u_{Q_{j}}(x) \chi_{Q_{j}}(x)$.

In order to prove (1), we take $R>1$ such that $Q \subset Q_{R}$, and decompose each $f \in L^{p}(w)$ as $f=f^{\prime}+f^{\prime \prime}$, where $f^{\prime \prime}=f \chi_{Q_{2 R}}$ and $f^{\prime}=f-f^{\prime \prime}$. Then, an elementary geometric argument shows that, for every $x \in Q$

$$
\begin{aligned}
M f^{\prime}(x) & \leqslant \sup _{h>R}\left|Q_{h}\right|^{-1} \int_{Q_{2 h}}\left|f^{\prime}\right| \\
& <\sup _{h>R} 2^{n}\left\|f^{\prime}\right\|_{L^{P(w)}}\left|Q_{2 h}\right|^{-1}\left(\int_{Q_{2 h} w^{-p^{\prime} / p}}\right)^{1 / p^{\prime}}<C_{R}\left\|f^{\prime}\right\|_{L^{P}(w)}
\end{aligned}
$$

so that we obtain

$$
\int_{Q}\left(M f^{\prime}\right)^{p}<|Q| C_{R}^{p} \int_{\mathbf{R}^{n}}\left|f^{\prime}\right|^{p_{w}} \quad\left(f \in L^{p}(w)\right)
$$

On the other hand, given scalars $\left(\alpha_{j}\right)$ and functions $\left(f_{j}\right)$ such that $\operatorname{supp}\left(f_{j}\right) \subset Q_{2 R}$, $\int\left|f_{j}\right|^{p_{w}}<1$, we use the $l^{p}$-valued extension of the weak type $(1,1)$ inequality for the maximal operator due to Fefferman and Stein [3] to get

$$
\begin{aligned}
& \left|\left\{x:\left(\sum_{j}\left|\alpha_{j} M f_{j}(x)\right|^{p}\right)^{1 / p}>\lambda\right\}\right|<A_{p} \lambda^{-1} \int_{Q_{2 R}}\left(\sum_{j}\left|\alpha_{j} f_{j}\right|^{p}\right)^{1 / p} d x \\
& \leqslant A_{p} \lambda^{-1}\left(\int \sum_{j}\left|\alpha_{j} f_{j}(x)\right|^{p} w(x) d x\right)^{1 / p}\left(\int_{Q_{2 R}} w^{-p^{\prime} / p}\right)^{1 / p^{\prime}}<A_{R, p} \lambda^{-1}\left(\sum_{j}\left|\alpha_{j}\right|^{p}\right)^{1 / p} .
\end{aligned}
$$


If $q<1$, Kolmogorov's inequality relating the $L^{q}$ norm with the weak- $L^{1}$ norm implies

$$
\int_{Q}\left(\sum_{j}\left|\alpha_{j} M f_{j}(x)\right|^{p}\right)^{q / p} d x \leqslant \frac{A_{R p}}{1-q}|Q|^{1-q}\left(\sum_{j}\left|\alpha_{j}\right|^{p}\right)^{q / p} .
$$

According to Maurey's factorization theorem [7, Theorem 2], there exists a measurable function $g$ such that

$$
\int_{Q}|g|^{r}<\infty, \quad \int_{Q}\left|\frac{M h(x)}{g(x)}\right|^{p} d x \leqslant 1 \quad\left(\frac{1}{r}=\frac{1}{q}-\frac{1}{p}\right)
$$

for every function $h$ supported in $Q_{2 R}$ with $\|h\|_{L^{p}(w)}<1$. Thus

$$
\int_{Q}\left(M f^{\prime \prime}(x)\right)^{p}|g(x)|^{-p} d x<\int_{\mathbf{R}^{n}}\left|f^{\prime \prime}\right|^{p} \quad\left(f \in L^{p}(w)\right) .
$$

From (2) and (3) we obtain (1) with $u_{Q}(x)=2^{1-p} \inf \left(|g(x)|^{-p},|Q|^{-1} C_{R}^{-p}\right)$. Since $u_{Q}^{-r / p} \in L^{1}(Q)$, and $r / p=q /(p-q)$ increases to $p^{\prime} / p$ as $q \rightarrow 1$, the last assertion of the theorem also follows.

The dual question, i.e., finding conditions on $u(x)$ so that $M$ is bounded from some $L^{p}(w)$ to $L^{p}(u)$, was also raised in [9]. A partial answer is contained in the following.

TheOREM B. Given $u(x)>0$ in $\mathbf{R}^{n}$ and $1<p<\infty$, in order that there exists $w(x)<\infty$ a.e. such that $M$ is bounded from $L^{p}(w)$ to $L^{p}(u)$, it is

(i) necessary that $u \in L_{\mathrm{loc}}^{1}$ and $\lim \sup _{R \rightarrow \infty}\left|Q_{R}\right|^{-1}\left(\int_{Q_{R}} u\right)^{1 / p}<\infty$,

(ii) sufficient that $u \in L_{\text {loc }}^{1}$ and, for some $q<p$, $\lim \sup _{R \rightarrow \infty}\left|Q_{R}\right|^{-1}\left(\int_{Q_{R}} u\right)^{1 / q}<\infty$.

Proof. If $M$ is bounded from $L^{p}(w)$ to $L^{p}(u)$, the pair $(u, w)$ belongs to $A_{p}$, and part (i) follows easily. The proof of (ii) depends on the following fact which will be obtained as a by-product of the results for singular integral operators:

[*] If $u \in L_{\text {loc }}^{1}$ and $\lim \sup _{R \rightarrow \infty}\left|Q_{R}\right|^{-1}\left(\int_{Q_{R}} u\right)^{1 / q}<\infty$, for every $r>q>1$ there exists $w(x)>0$ such that $(u, w) \in A_{r}$.

Using [*] with $q<r<p$ we see that $M$ is bounded from $L^{r}(w)$ to weak- $L^{r}(u)$, and since it is bounded on $L^{\infty}$ we only have to interpolate by the Marcinkiewicz theorem.

3. Boundedness of singular integrals. By a singular integral operator (s.i.o.) in $\mathbf{R}^{n}$ we shall mean an operator of the form

$$
T f(x)=K * f(x)=\text { p.v. } \int K(y) f(x-y) d y
$$

with the kernel $K$ satisfying the conditions

$$
\begin{aligned}
|\hat{K}(x)| & \leqslant B, \\
|K(x)| & <B\|x\|^{-n}, \\
|K(x-y)-K(x)| & \leqslant B\|y\| /\|x\|^{n+1} \text { when }\|y\|<\|x\| / 2,
\end{aligned}
$$


where $\|\cdot\|$ stands for Euclidean norm in $\mathbf{R}^{n}$. The least constant $B$ for which (4), (5) and (6) hold will be denoted by $B(T)$. The simplest examples of such operators are the Riesz transforms

$$
R_{j} f=K_{j} * f, \quad K_{j}(x)=c_{n} x_{j} /\|x\|^{n+1} \quad(j=1,2, \ldots, n)
$$

where $c_{n}=\pi^{-(n+1) / 2} \Gamma((n+1) / 2)($ see $[11])$.

TheOREM C. Given $w(x)>0$ in $\mathbf{R}^{n}$ and $1<p<\infty$, the following conditions are equivalent:

(a) There exists $u(x)>0$ in $\mathbf{R}^{n}$ such that, for every singular integral operator of the type described above

$$
\int|T f(x)|^{p} u(x) d x<B(T) \int|f(x)|^{p} w(x) d x \quad\left(f \in L^{p}(w)\right) .
$$

(b) $w^{-p^{\prime} / p} \in L_{\text {loc }}^{1}$ and $\int_{\mathbf{R}^{n} w}(x)^{-p^{\prime} / p}(1+\|x\|)^{-n p^{\prime}} d x<\infty$.

(c) The Riesz transforms are continuous operators from $L^{p}(w)$ to $L^{0}\left(\mathbf{R}^{n}\right)$.

Moreover, if any of these conditions hold and $s<p^{\prime} / p, u(x)$ can be obtained in (a) such that $u^{-s} \in L_{\text {loc }}^{1}$.

Proof. (a) implies (c). This is obvious because convergence in $L^{p}(u)$ (with $u(x)>0$ everywhere in $\mathbf{R}^{n}$ ) implies local convergence in measure. In fact, if $f_{j} \rightarrow 0$ (in $L^{p}(u)$ ) and $m_{u}$ denotes the measure $d m_{u}(x)=u(x) d x$,

$$
m_{u}\left(\left\{x:\left|f_{j}(x)\right|>\lambda\right\}\right)<\lambda^{-p}\left\|f_{j}\right\|_{L(u)}^{p} \rightarrow 0
$$

for every $\lambda>0$, and since $u^{-1} \in L^{1}\left(Q, m_{u}\right)$ for every cube $Q$

$$
\left|\left\{x \in Q:\left|f_{j}(x)\right|>\lambda\right\}\right|=\int_{\left\{\left|f_{j}\right|>\lambda\right\} \cap Q} u^{-1} d m_{u} \rightarrow 0 .
$$

(c) implies (b). Since $R=\sum_{j=1}^{n} R_{j}$ is continuous in measure in $L^{p}(w)$, if we fix our attention on the unit ball $B=\left\{x \in R^{n}:\|x\|<1\right\}$, there exists $\lambda_{0}>0$ big enough so that

$$
\left|\left\{x \in B:|\operatorname{Rg}(x)|>\lambda_{0}\|g\|_{L^{P(w)}}\right\}\right|<2^{-n}|B|
$$

for all $g \in L^{P}(w)$. Let $P=\left\{x \in \mathbf{R}^{n}: x_{1}>0, x_{2}>0, \ldots, x_{n}>0\right\}$ be the first "quadrant" in $\mathbf{R}^{n}$. If $f \in L^{p}(w)$ and $x \in(-P) \cap B$,

$$
\begin{aligned}
\left|R\left(|f| \chi_{P}\right)(x)\right| & =\sum_{j=1}^{n} c_{n} \int_{P}|f(y)|\left(x_{j}-y_{j}\right)\|x-y\|^{-n-1} d y \\
& =c_{n} \int_{P}|f(y)|\left(\sum_{j=1}^{n}\left|x_{j}-y_{j}\right|\right)\|x-y\|^{-n-1} d y>c_{n} \int_{P}|f(y)|\|x-y\|^{-n} d y \\
& \geqslant c_{n} \int_{P}|f(y)|(1+\|y\|)^{-n} d y .
\end{aligned}
$$

Since $|(-P) \cap B|=2^{-n}|B|$, (7) implies

$$
c_{n} \int_{P}|f(y)|(1+\|y\|)^{-n} d y<\lambda_{0}\left\|f \chi_{P}\right\|_{L^{P}(w)}
$$


By the same argument, (8) holds if we replace $P$ by any other of the $2^{n}$ "quadrants" in $\mathbf{R}^{n}$. Therefore

$$
\int_{\mathbf{R}^{n}}|f(y)|(1+\|y\|)^{-n} d y<2^{n / p^{\prime}} c_{n}^{-1} \lambda_{0}\|f\|_{L^{P(w)}}
$$

By writing the integrand as $|f(y)|(1+\|y\|)^{-n} w(y)^{-1} w(y) d y$, we see that the function $w(y)^{-1}(1+\|y\|)^{-n}$ belongs to $L^{p^{\prime}}(w)$. This proves (b).

(b) implies (a). Fix a cube $Q$ and take $R>1$ such that $Q \subset\{x:\|x\| \leqslant R\}$. As in Theorem A, it will suffice to find a constant $C>0$ and a function $v(x)>0$ on $Q$ with $v^{-s} \in L^{1}(Q)$ (where $s<p^{\prime} / p$ is given) such that, for every s.i.o. $T$ with $B(T) \leqslant 1$, the following inequalities hold:

$$
\begin{gathered}
\int_{Q}|T f(x)|^{p} v(x) d x \leqslant\|f\|_{L^{p}(w)}^{p} \quad \text { when } \operatorname{supp}(f) \subset\{x:\|x\| \leqslant 2 R\}, \\
\sup _{x \in Q}|T f(x)| \leqslant C\|f\|_{L^{P(w)}} \quad \text { when } \operatorname{supp}(f) \subset\{x:\|x\|>2 R\} .
\end{gathered}
$$

To prove (10) we take $C=\frac{3}{2}\left(\int_{\|y\| \geq 1} w(y)^{-p^{\prime} / p}\|y\|^{-n p^{\prime}} d y\right)^{1 / p^{\prime}}$. Then, if $f$ is supported in $\{x:\|x\| \geqslant 2 R\}$ and $B(T) \leqslant 1$, we have by (5) and (6)

$$
\begin{aligned}
\sup _{x \in Q}|T f(x)| & \leqslant \sup _{x \in Q} \int_{\|y\|>2 R}|f(y)||K(x-y)| d y \\
& \leqslant \sup _{x \in Q} \int_{\|y\|>2 R}|f(y)|\left(\|x\|\|y\|^{-n-1}+|K(y)|\right) d y \\
& \leqslant \frac{3}{2} \int_{\|y\|>2 R}|f(y)|\|y\|^{-n} d y \leqslant C\|f\|_{L P(w)}
\end{aligned}
$$

The proof of (9) depends on the vector valued inequalites for singular integrals due to Benedek, Calderón and Panzone [1]. Given a sequence of s.i.o. $\left(T_{j}\right)_{1}^{\infty}$ with $B\left(T_{j}\right) \leqslant 1$, the operator $\tilde{T}$ defined on $l^{p}$-valued functions by $\tilde{T}\left(f_{1}, f_{2}, \ldots, f_{j}, \cdots\right)$ $=\left(T_{1} f_{1}, T_{2} f_{2}, \ldots, T_{j} f_{j}, \ldots\right)$ satisfies the hypothesis of [1, Theorem 1], and therefore, it is of weak type $(1,1)$, i.e.

$$
\left|\left\{x \in \mathbf{R}^{n}:\left(\sum_{j}\left|T_{j} f_{j}(x)\right|^{p}\right)^{1 / p}>\lambda\right\}\right|<A_{p} \lambda^{-1}\left\|\left(\sum_{j}\left|f_{j}\right|^{p}\right)^{1 / p}\right\|_{1}
$$

with $A_{p}$ depending only on $p$ (and not on the particular sequence of operators $\left(T_{j}\right)$, provided that $B\left(T_{j}\right) \leqslant 1$ ). By the same argument as Theorem A (e.g. Kolmogorov's inequality and Maurey's Theorem 2 of [7]) we obtain a function $g \in L^{r}(Q)$, with $\frac{1}{r}=\frac{1}{q}-\frac{1}{p}$ and $q<1$ arbitrarily close to 1 , such that $\int_{Q}|h(x) / g(x)|^{p} d x<1$ for any function $h$ in the family

$$
\mathscr{F}=\left\{T f \mid T \text { s.i.o. with } B(T) \leqslant 1,\|f\|_{L^{P(w)}} \leqslant 1, \operatorname{supp}(f) \subset\{x:\|x\|<2 R\}\right\} .
$$

This proves (9) with $v(x)=|g(x)|^{-p}$, and taking $q$ so that $r / p=q /(p-q)=s$, it follows that $v^{-s} \in L^{1}(Q)$.

Since every s.i.o. $T$ is selfadjoint, $T$ is bounded from $L^{P}(w)$ to $L^{P}(u)$ if and only if it is bounded (with the same norm) from $L^{p^{\prime}}\left(u^{-p^{\prime} / p}\right)$ to $L^{p^{\prime}}\left(w^{-p^{\prime} / p}\right)$ (see [10] for the 
simple proof of this fact). Therefore, Theoerem $\mathrm{C}$ already gives us the complete answer of the dual question for s.i.o.

TheOREM D. Given $u(x)>0$ in $\mathbf{R}^{n}$ and $1<p<\infty$, the following conditions are equivalent:

(a) There exists $w(x)<\infty$ a.e. such that, for every singular integral operator of the type considered here

$$
\int|T f(x)|^{p} u(x) d x \leqslant B(T) \int|f(x)|^{p} w(x) d x \quad\left(f \in L^{p}(w)\right) .
$$

(b) $u \in L_{\text {loc }}^{1}$ and $\int_{\mathbf{R}^{n}} u(x)(1+\|x\|)^{-n p} d x<\infty$.

(c) There exists $w(x)<\infty$ a.e. such that the Riesz transforms are bounded from $L^{p}(w)$ to $L^{p}(u)$.

Moreover, given $s<1, w(x)$ in (a) and (c) can be obtained such that $w^{s} \in L_{\text {loc }}^{1}$

At this point, the fact needed in the proof of Theorem $B$ is easy to obtain.

Proof of [*]. We assume that $u \in L_{\text {loc }}^{1}, u>0$ and $h(t)=\int_{\|x\|<t} u<C t^{n q}(t>$ 1). If $q<r$, by using polar coordinates and integration by parts

$$
\begin{aligned}
\int_{\mathbf{R}^{n}} u(x)(1+\|x\|)^{-n r} d x & =\int_{0}^{\infty}(1+t)^{-n r} t^{n-1} d t \int_{\left\|x^{\prime}\right\|=1} u\left(t x^{\prime}\right) d \sigma\left(x^{\prime}\right) \\
& =\int_{0}^{\infty} h^{\prime}(t)(1+t)^{-n r} d t=n r \int_{0}^{\infty} h(t)(1+t)^{-n r-1} d t<\infty
\end{aligned}
$$

By Theorem D, there exists $w(x)<\infty$ a.e. such that the Riesz transforms are bounded from $L^{r}(w)$ to $L^{r}(u)$, and this implies $(u, w) \in A_{r}$ (see [2, 9]).

The proofs of Theorems A, B, C, D work also in the periodic case (and are even simpler because there is no limitation at infinity for the weights). In particular, for the torus $\mathbf{T} \cong[0,1)$, if we denote by $\tilde{f}$ the conjugate function of $f \in L^{1}(T)$, we ask for weights $u(x), w(x)$ such that

$$
\int_{\mathbf{T}}|\tilde{f}|^{p_{u}} \leqslant \int_{\mathbf{T}}|f|^{p_{w}} \quad(f \text { trigonometric polynomial })
$$

Corollary. (i) Given $w(x)>0$ in $\mathbf{T}$ and $1<p<\infty$, (11) holds for some $u(x)>0$ if and only if $w^{-p^{\prime} / p} \in L^{1}(\mathbf{T})$. In this case, and if $s<p^{\prime} / p$ is given, $u$ can be found such that $u^{-s} \in L^{1}(\mathbf{T})$.

(ii) Given $u(x)>0$ in $\mathrm{T}$ and $1<p<\infty$, (11) holds for some $w(x)<\infty$ a.e. if and only if $u \in L^{1}(\mathrm{~T})$. In this case, and if $s<1$ is given, $w$ can be found such that $w^{s} \in L^{1}(\mathrm{~T})$.

For $p=2$, (i) has been proved by P. Koosis [6], who obtains $u(x)$ such that $\log u \in L^{1}(\mathbf{T})$. The corollary is also true for the inequality (11) with $M f$ (maximal function of $f \in L^{1}(T)$ ) instead of $\tilde{f}$ (part (ii) is well known in this case; see [3, Lemma 1]).

Acknowledgement. I wish to express my thanks to G. Pisier for an enlightening discussion about factorization theorems while this paper was in preparation. I also thank the referee for his criticism and helpful suggestions. 
ADDED IN PROOF. L. Carleson and P. Jones have obtained essentially the same results of Theorems $\mathrm{A}$ and $\mathrm{C}$ by a somewhat different method (Mittag-Leffler Institute, Report No. 2, 1981).

\section{REFERENCES}

1. A. Benedek, A. P. Calderon and R. Panzone, Convolution operators on Banach space valued functions, Proc. Nat. Acad. Sci. U.S.A. 48 (1962), 356-365.

2. R. R. Coifman and C. Fefferman, Weighted norm inequalities for maximal functions and singular integrals, Studia Math. 51 (1974), 241-250.

3. C. Fefferman and E. M. Stein, Some maximal inequalities, Amer. J. Math. 1 (1971), 107-115.

4. A. M. Garsia, Topics in almost everywhere convergence, Markham, Chicago, Ill., 1970.

5. J. E. Gilbert, Nikishin-Stein theory and factorization with applications, Proc. Sympos. Pure Math., vol. 35, part 2, Amer. Math. Soc., Providence, R. I., 1979, pp. 233-267.

6. P. Koosis, Moyennes quadratiques pondérées de fonctions périodiques et de leurs conjuguées harmoniques, C. R. Acad. Sci. Paris Sér. A 291 (1980), 255-257.

7. B. Maurey, Theorèmes de factorisation pour les opérateurs linéaires à valeurs dans les espaces $L^{p}$, Astérisque, no. 11, Soc. Math. de France, 1974.

8. B. Muckenhoupt, Weighted norm inequalities for the Hardy maximal function, Trans. Amer. Math. Soc. 165 (1972), 207-226.

9. __ Weighted norm inequalities for classical operators, Proc. Sympos. Pure Math., vol. 35, part 1, Amer. Math. Soc., Providence, R. I.,' 1979, pp. 69-83.

10. J. L. Rubio de Francia, Vector valued inequalities for operators in $L^{p}$ spaces, Bull. London Math. Soc. 12 (1980), 211-215.

11. E. M. Stein, Singular integrals and differentiability properties of functions, Princeton Univ. Press, Princeton, N. J., 1980.

facultad de Ciencias, University of Zaragoza, Zaragoza, Spain 